\title{
1 3D bioprinting of co-cultured osteogenic spheroids for bone tissue fabrication
}

2 Dong Nyoung Heo, ${ }^{\mathrm{a}, \mathrm{b}, \mathrm{c}, 1}$, Bugra Ayan, ${ }^{\mathrm{a}, \mathrm{b}, 1}$, Madhuri Dey ${ }^{\mathrm{b}, \mathrm{d}}$, Dishary Banerjee, ${ }^{\mathrm{a}, \mathrm{b}}$, Hwabok

3 Wee, ${ }^{\mathrm{e}}$ Gregory S. Lewis, ${ }^{\mathrm{e}}$ Ibrahim T. Ozbolat ${ }^{* a, b, f, g, h}$

4

5 a Department of Engineering Science and Mechanics Department, Penn State University,

6 University Park, PA 16802, USA

$7{ }^{\mathrm{b}}$ The Huck Institutes of the Life Sciences, Penn State University, University Park, PA 16802,

$8 \quad U S A$

$9{ }^{\mathrm{c}}$ Department of Dental Materials, School of Dentistry, Kyung Hee University, 26

10 Kyungheedae-ro, Dongdaemun-gu, Seoul 02447, Republic of Korea

$11{ }^{d}$ Department of Chemistry, Penn State University, University Park, PA 16802, USA

12 e Department of Orthopedics and Rehabilitation, Penn State College of Medicine, Hershey,

13 PA 17033, USA

$14{ }^{\mathrm{f}}$ Biomedical Engineering Department, Penn State University, University Park, PA 16802,

$15 \quad U S A$

$16{ }^{\mathrm{g}}$ Materials Research Institute, Penn State University, University Park, PA 16802, USA

$17{ }^{\mathrm{h}}$ Department of Neurosurgery, Penn State College of Medicine, Hershey, PA 17033, USA

$18{ }^{1}$ These authors contributed equally to this work

19 * Correspondence to Ibrahim T. Ozbolat, Ph. D.

20 Engineering Science and Mechanics Department, Biomedical Engineering Department, The

21 Huck Institutes of the Life Sciences, Materials Research Institute

22 The Pennsylvania State University

23 W313 Millennium Science Complex, University Park, PA 16802, USA

24 Contact: 1-814-863-5819/ito1@ psu.edu (Ibrahim T. Ozbolat). 


\section{Abstract}

26 Conventional top-down approaches in tissue engineering involving cell seeding on scaffolds

27 have been widely used in bone engineering applications. However, scaffold-based bone tissue

28 constructs have had limited clinical translation due to constrains in supporting scaffolds,

29 minimal flexibility in tuning scaffold degradation, and low achievable cell seeding density as

30 compared with native bone tissue. Here, we demonstrate a pragmatic and scalable bottom-up

31 method, inspired from embryonic developmental biology, to build three-dimensional (3D)

32 scaffold-free constructs using spheroids as building blocks. Human umbilical vein endothelial

33 cells (HUVECs) were introduced to human mesenchymal stem cells (hMSCs)

34 (hMSC/HUVEC) and spheroids were fabricated by an aggregate culture system. Bone tissue

35 was generated by induction of osteogenic differentiation in hMSC/HUVEC spheroids for 10

36 days, with enhanced osteogenic differentiation and cell viability in the core of the spheroids

37 compared to hMSC-only spheroids. Aspiration-assisted bioprinting (AAB) is a new

38 bioprinting technique which allows precise positioning of spheroids (11\% with respect to the

39 spheroid diameter) by employing aspiration to lift individual spheroids and bioprint them

40 onto a hydrogel. AAB facilitated bioprinting of scaffold-free bone tissue constructs using the

41 pre-differentiated hMSC/HUVEC spheroids. These constructs demonstrated negligible

42 changes in their shape for two days after bioprinting owing to the reduced proliferative

43 potential of differentiated stem cells. Bioprinted bone tissues showed interconnectivity with

44 actin-filament formation and high expression of osteogenic and endothelial-specific gene

45 factors. This study thus presents a viable approach for 3D bioprinting of complex-shaped

46 geometries using spheroids as building blocks, which can be used for various applications

47 including but not limited to, tissue engineering, organ-on-a-chip and microfluidic devices, 48 drug screening and, disease modeling. 
bioRxiv preprint doi: https://doi.org/10.1101/2020.06.16.155143; this version posted June 17, 2020. The copyright holder for this preprint (which was not certified by peer review) is the author/funder. All rights reserved. No reuse allowed without permission.

49 Keywords: biofabrication, 3D bioprinting, aspiration-assisted bioprinting, osteogenic

50 spheroids, bone tissue regeneration 


\section{1. Introduction}

52 Bone is a highly vascularized dynamic tissue, observed in a variety of shapes and sizes in

53 the body. The skeleton serves crucial roles such as supporting the framework for the body

54 and protecting vital organs [1,2]. Large bone defects cannot self-regenerate regenerate, and

55 can be due to trauma, fracture nonunion, infection, bone tumor resections, and

56 removal/revision of joint replacements and other implant [3-5]. There is thus a substantial

57 demand for engineered constructs to restore and regenerate the diseased/excised part of the

58 bone tissue $[4,6,7]$. To-date, significant progress has been made using three-dimensional (3D)

59 bioprinting of living cells and tissues to recapitulate the bone tissue [8]. Most of these

60 bioprinting approaches rely on scaffold-based techniques where biodegradable hydrogels are

61 seeded or combined with mature osteoblasts or osteogenically-committed stem-cells $[6,9,10]$.

62 These scaffolds can provide mechanical support, serve as a template for cell attachment and

63 facilitate a conducive environment for cellular activities [3,6]. However, degradation of

64 scaffolds, limited cell density compared to native-tissues and limited communication among

65 cells are some of the major drawbacks in scaffold-based approaches [11].

66 Scaffold-free bone tissue engineering pose a promising alternative to $3 \mathrm{D}$ bioprinting

67 approaches [12]. Stem-cell derived aggregates, in the form of spheroids, are considered as

68 building blocks for scaffold-free bioprinting and mimic the complex morphology and

69 physiology of native tissue by inducing cross-talk among cells and cell-extracellular cell

70 matrix (ECM) interactions [13,14]. In addition, osteogenic differentiation is observed to

71 increase due to stronger integrin-ECM interaction caused by the presence of both stroma and

72 structure within spheroids [15]. However, non-uniform bone tissue regeneration owing to the

73 non-homogeneous oxygen diffusion across the entire spheroid domain, especially to the core

74 of spheroids [15-17] forms the major roadblock in successful usage of spheroids in 3D 
75 bioprinting applications. 2D co-culture systems with mature osteoblasts or stem cell derived

76 osteogenic progenitor cells with cells from endothelial lineage have shown potential to

77 address this limitation by enhanced secretion of endothelial cell-mediated paracrine factors

78 secretion under hypoxia [18]. In this study, we intended to exploit this potential of endothelial

79 cells and investigate their role in $3 \mathrm{D}$ on osteogenic differentiation. We achieved this by the

80 co-culture of a minimal number of human umbilical vein endothelial cells (HUVEC) in

81 human mesenchymal stem cells (hMSC) spheroids, and by induction of osteogenesis with

82 bone regeneration across the entire domain of spheroids.

83 Spheroids have been utilized in successful biofabrication of functional bone tissue substitutes

84 [19-22]. Although several approaches have been presented in the literature for bioprinting of

85 spheroids, most of these suffer from poor spatial control in $3 \mathrm{D}$, significant damages to

86 spheroids with loss of viability and structural integrity, poor repeatability of the process while

87 using spheroids that are non-uniform in size, inability to form complex 3D shapes, inability to

88 maintain designed shape due to cell-mediated compaction post-bioprinting, and lack of

89 scalability for translation from bench to bedside [19-21]. Aspiration-assisted bioprinting

90 (AAB), a newly developed technique by our group, bioprints spheroids in 3D space using the

91 power of aspiration forces. AAB leverages the fact that spheroids can be formed from diverse

92 cell types at high densities [22]. When using human stem cells, these spheroids can

93 recapitulate aspects of embryonic development to self-assemble [21] into various organs.

94 Using this technique, in this study, we have demonstrated that spheroids with viscoelastic

95 properties can be lifted by aspiration forces, and then positioned precisely (11\% with respect

96 to the spheroid diameter) into a hydrogel substrate, circumventing the limitations of the other

97 spheroid bioprinting techniques available. 
98 Here, co-culture of hMSCs and HUVEC spheroids were utilized to enhance cellular

99 function, osteogenic differentiation, calcium deposition, and cell viability. After

100 biofabrication of spheroids, two different strategies were demonstrated for the bioprinting of

101 bone tissue using hMSC/HUVEC and pre-differentiated hMSC/HUVEC spheroids.

102 Compaction of spheroids leading to significant changes in geometry of tissue constructs

103 compared to the desired has been a common issue post bioprinting, and hence, we utilize 3D

104 bioprinting of pre-differentiated hMSC/HUVEC spheroid to minimize shape changes. Post-

105 bioprinting, we showed that we were able to control the shape of the bioprinted construct,

106 using pre-differentiated hMSC/HUVEC spheroids. In this study, thus, we attempted to

107 address major limitations of spheroid bioprinting by bioprinting spheroids using $\mathrm{AAB}$,

108 fabricating complex-shaped bone tissue constructs, engineering the spheroids in a way to

109 induce osteogenesis across the entire spheroid domain, and controlling osteogenic induction

110 timelines before and after bioprinting in order to reduce spheroid compaction and increase

111 retention of geometry of the tissue constructs.

112

\section{2. Experiment Section}

\section{2.1. Cell culture}

115 HMSCs (Lonza, Walkersville, MD) and HUVECs (Lonza) were used to fabricate of 3D

116 cellular spheroids. hMSCs were cultured in all-in-one ready-to-use hMSC growth medium

117 (Cell Applications, INC., San Diego, CA). HUVECs or tdTomato ${ }^{+}$HUVECs were cultured in

118 MCDB 131 medium (Corning, New York, NY) supplemented with $10 \%$ fetal bovine serum

119 (Corning, New York, NY), 2 mM glutamine (Thermo Fisher Scientific, Waltham, MA), 1\%

120 penicillin/streptomycin (Corning, New York, NY), $4.5 \mathrm{ug} / \mathrm{mL}$ bovine brain extract (Lonza,

121 Walkersville, MD), 10 unit/mL heparin (Sigma-Aldrich, St. Louis, MO), and $1.86 \mathrm{mg} / \mathrm{mL}$ 
122 endothelial cell growth supplement (Sigma-Aldrich, St. Louis, MO). Cells passages from

123 three through seven were used for both hMSCs and HUVECs. The cells were expanded at 37

$124{ }^{\circ} \mathrm{C}$ with $5 \% \mathrm{CO}_{2}$ in a humidified sterile incubator.

\section{$126 \quad$ 2.2. Spheroid fabrication}

127 hMSCs and HUVECs were harvested with trypsin and collected by centrifugation at 1600

$128 \mathrm{rpm}$ for $5 \mathrm{~min}$ for the fabrication of the spheroids. hMSCs were reconstituted to $2.5 \times 10^{5}$

129 cells per ml, with various mixing ratios of HUVECs $(8,15$, and $25 \%)$, referred as 92:8, 85:15

130 and 75:25 hMSC/HUVEC respectively. $200 \mu \mathrm{l}$ of cell suspension was seeded into each well

131 of 96-well plates with U-bottom, cell-repellent surface (Greiner bio-one, Frickenhausen,

132 Germany) to achieve $\sim 50,000$ cells/spheroid. Cells were maintained in hybrid growth media

133 (75\% MSC and 25\% HUVEC growth media) to allow compaction at $37{ }^{\circ} \mathrm{C}$. HMSC-only

134 spheroids were fabricated similarly and used as control to understand the functionality of

135 HUVECs in the spheroids. Osteogenic differentiation was induced in hMSC-only spheroids

136 and hMSC/HUVEC spheroids by cultured in human osteogenic differentiation medium (Cell

137 Applications, INC., San Diego, CA) for 10 days. The cell medium was changed every three 138 days.

\section{$140 \quad$ 2.3. Characterization of spheroids}

141 The diameters of hMSC-only and hMSC/HUVEC spheroids were imaged and further 142 quantified using EVOS FL cell imaging system (Thermo Fisher Scientific, Waltham, MA) 143 and ImageJ (NIH Freeware) respectively from each of days, 1 to 5 post-seeding. Cell viability 144 was confirmed using a calcein-AM/ethidium homodimer-1 (EthD-1) LIVE/DEAD assay kit 145 (Invitrogen, Carlsbad, CA) [22]. Briefly, five days after incubation, spheroids were washed 
146 with Dulbecco's phosphate-buffered saline (DPBS), stained with $2 \mu \mathrm{M}$ calcein AM and $4 \mu \mathrm{M}$

147 EthD-1 solutions for $40 \mathrm{~min}$, and then imaged as described before.

148 To evaluate the cellular organization of hMSC-only and hMSC/HUVEC spheroids, spheroids

149 were fixed in $10 \%$ formalin, embedded in paraffin using a Leica TP 1020 automatic tissue

150 processor (Leica, Wetzlar, Germany) and sectioned with 10- $\mu \mathrm{m}$ thickness. Cross-sectioned

151 samples were stained with hematoxylin and eosin (H\&E) using a Leica Autostainer XL

152 (Leica, Wetzlar, Germany) and then imaged using optical microscopy. In addition, the

153 cytoskeletal organization was confirmed with actin staining. Cross-sectioned samples were

154 permeabilized in $0.1 \%$ Triton $\mathrm{X}-100$ for $30 \mathrm{~min}$, blocked with $2.5 \%$ normal goat serum

155 (NGS) for $60 \mathrm{~min}$ at room temperature and incubated with ActinGreen ${ }^{\mathrm{TM}} 488$ (1:1000 in

156 2.5\% NGS) and 4',6-Diamidino-2-Phenylindole (DAPI; 1:1000 in 2.5\% NGS) for $60 \mathrm{~min}$.

157 Then, the stained samples were washed thrice with DPBS and observed under an optical 158 microscope.

\section{2.4. Surface tension measurements}

161 The surface tension of hMSC-only and hMSC/HUVEC spheroids was measured using a 162 micropipette aspiration technique, as previously described [23]. The customized 163 micropipettes were prepared from borosilicate Pasteur pipettes (vWR, 14673-043, Radnor, 164 PA) on a P1000 Flaming/Brown micropipette puller (Sutter Instrument, Novato, CA). 165 Parameters for heat, pull, velocity, time, and pressure were set at 575, 0, 5, 100, and 50, 166 respectively according to our recent study [22]. All spheroid types were aspirated by a 167 customized glass pipette and monitored via an STC-MC33USB monochromatic camera 168 (Sentech, Japan) equipped with 1-61448 and 1-61449 adaptor tubes (Navitar, Rochester, 169 NY). 
$171 \quad 2.5$ Quantitative real-time polymerase chain reaction (real-time PCR)

172 Real-time polymerase chain reaction (RT-qPCR) was performed after five days in growth

173 media, and an additional 10 days in osteogenic media to evaluate the gene expression profiles

174 of hMSC-only and hMSC/HUVEC spheroids. The total RNA of hMSC-only and

175 hMSC/HUVEC spheroids was isolated using a RNeasy Plus Mini Kit (Qiagen, Germantown,

176 MD) according to the manufacturer's instructions and quantified using a Nanodrop ND-1000

177 Spectrophotomer (Thermo Scientific, Wilmington, DE). The primers of the measured mRNA

178 genes were as follows: OCT4 (forward, GCA GCG ACT ATG CAC AAC GA and reverse,

179 CCA GAG TGG TGA CGG AGA CA), PECAM-1 (forward, TAA TAC AAC ATC CAC

180 GAG GGT CC and reverse, ACA AAA TTG CTT GCT AAA GAA GTG G), HIF1A

181 (forward, CCA GTT AGG TTC CTT CGA TCA GT and reverse, TTT GAG GAC TTG CGC

182 TTT CA), COL6A1 (forward, CCT GGA GGG CTA CAA GGA A and reverse, GTG CTT

183 GGC CTC GTT CAC), COL1 (forward, ATG ACT ATG AGT ATG GGG AAG CA and

184 reverse, TGG GTC CCT CTG TTA CAC TTT), ALP (forward, AGC TGA ACA GGA ACA

185 ACG TGA and reverse, CTT CAT GGT GCC CGT GGT C), BSP (forward, AAC GAA

186 GAA AGC GAA GCA GAA and reverse, TCT GCC TCT GTG CTG TTG). RT-qPCR was

187 analyzed using SsoFast ${ }^{\mathrm{TM}}$ EvaGreen $^{\circledR}$ Supermix (Bio-Rad, Hercules, CA) and all values were

188 normalized by a house-keeping gene GAPDH. Threshold cycle values were calculated using

189 a comparative cycle threshold method. The fold-change of hMSC-only was set at 1-fold, and

190 the ratio of the normalized fold-change was calculated based on the standard condition.

191

$192 \quad 2.63 D$ bioprinting of spheroids 
193 AAB system was used to bioprint hMSC-only and hMSC/HUVEC spheroids as previously 194 described [22]. AAB system was equipped with microvalves (INKX0517500A, Lee

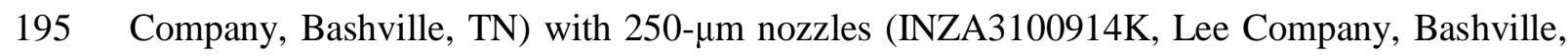
196 TN) and a portable ultrasonic humidifier (CZHD20, Comfort Zone, China). 1g of sodium 197 alginate (Sigma-Aldrich, UK) was dissolved in $100 \mathrm{ml}$ deionized (DI) water to prepare $1 \%$ 198 (w/v) sodium alginate as a sacrificial material. In order to generate alginate droplets using the 199 microvalve dispenser, dwell time of $700 \mu$ s was utilized with positive back pressure of $\sim 103$ $200 \mathrm{kPa}$. Calcium chloride $\left(\mathrm{CaCl}_{2}\right)$ solution was prepared by dissolving $4 \%(\mathrm{w} / \mathrm{v})\left(\mathrm{CaCl}_{2}(\mathrm{Sigma}-\right.$ 201 Aldrich, St. Louis, MO) in DI water. Sodium alginate was dispensed on the glass substrate 202 using microvalves, then the aerosol form of $\mathrm{CaCl}_{2}$ was utilized to crosslink sodium alginate 203 partially. Spheroids were collected into $1.5 \mathrm{ml}$ conical tubes and transferred to the bioprinting 204 platform. Afterwards, the top portion of the conical tubes were cut by a scissor. A customized 205 glass pipette $(\sim 80 \mu \mathrm{m}$ in diameter $)$ was dipped into a conical tube and air pressure of 25 $206 \mathrm{mmHg}$ was applied to lift spheroids. Critical lifting pressure was determined theoretically 207 from the mean radius of spheroids, surface tension coefficient of cell media and air interface, 208 and the dynamic contact angle at three phases (air, spheroid, and cell media) [22] to pick 209 spheroids. hMSC-only and hMSC/HUVEC spheroids were lifted from cell media with 210 backpressure of 43 and $52 \mathrm{mmHg}$, respectively. After the fabrication of complex-shaped 211 arrangements, the bioprinted constructs were overlaid with alginate and crosslinked with the 212 aerosol form. The whole construct was then transferred to a Petri dish and cultured with 213 tissue-specific cell media. 4\% (w/v) sodium citrate solution was added and agitated gently to 214 remove alginate. 
$216 \quad 2.7$ Characterization of osteogenic differentiation by immunocytochemistry and alizarin

\section{7 red S staining}

218 To visualize the morphologies of osteogenically differentiating spheroids, spheroids cultured

219 in osteogenic differentiation medium were stained with runt-related transcription factor 2

220 (RUNX2) and CD31. After osteogenic differentiation for 10 days, hMSC-only and

221 hMSC/HUVEC spheroids were cross-sectioned, permeabilized in $0.2 \%$ Triton X-100 for 30

222 min and blocked with $2.5 \%$ NGS for $1 \mathrm{~h}$. Then, the samples were incubated with mouse anti-

223 RUNX2 primary antibody (1:100 in $2.5 \%$ NGS) and rabbit anti-CD31 primary antibody

224 (1:100 in $2.5 \%$ NGS) for $60 \mathrm{~min}$ at room temperature, washed three times with DPBS, and

225 incubated with goat anti-mouse Alexa Fluor 488 secondary antibody (1:250 in 2.5\% NGS),

226 goat anti-rabbit Alexa Fluor 647 secondary antibody (1:250 in 2.5\% NGS), rhodamine-

227 phalloidin (1:250 in $2.5 \%$ NGS) and DAPI (1:1000 in $2.5 \%$ NGS) for 60 min at $4{ }^{\circ} \mathrm{C}$. The

228 stained samples were then washed three times with DPBS and imaged using an Olympus

229 FV10i-LIV Confocal Laser Scanning Microscope (Olympus America Inc., Melville, NY).

230 The calcium deposition was visualized by staining cross-sectioned slides with a $2 \%$ alizarin

231 red S staining solution for $10 \mathrm{~min}$ at room temperature. Stained samples were washed three

232 times with DI water and imaged using optical microscopy.

\section{$234 \quad 2.8$ Micro-computed tomography $(\mu \mathrm{CT})$ measurements}

$235 \mu \mathrm{CT}$ scanner (VivaCT 40, Scanco Medical, Switzerland) was used with $10.5 \mu \mathrm{m}$ voxel 236 resolution, $55 \mathrm{kV}$ energy, $145 \mu \mathrm{A}$ intensity, $21.5 \mathrm{~mm}$ diameter field-of-view, and $300 \mathrm{~ms}$ 237 integration time to evaluate the mineralization of spheroids in bioprinted tissues. The samples 238 were placed inside the $\mu \mathrm{CT}$ scanner and scanned. DICOM files were processed in Avizo 239 software (FEI Company, Hillsboro, OR). A hydroxyapatite (HA) phantom (Micro-CT HA, 
240 QRM, Germany) was included in each scan to generate a standard curve to convert

241 Hounsfield units to $\mathrm{mgHA} / \mathrm{ccm}$ [24]. Images were processed with a Gaussian smoothing filter

242 (sigma 0.9) to reduce noise, and a threshold of $200 \mathrm{mgHA} / \mathrm{ccm}$ was used to visualize

243 mineralized spheroids and quantify mineralized volume.

\section{$245 \quad 2.9$ Statistical analysis}

246 All values are presented as mean $( \pm)$ standard deviation. Multiple comparisons were analyzed 247 using a one-way analysis of variance (ANOVA) followed by Tukey's multiple comparison 248 test. The differences with p-values $(* p<0.05, * * p<0.01)$ were considered statistically

249 significant. All statistical analysis was performed by Statistical Product and Service Solutions 250 software (SPSS, IBM, Armonk, NY).

\section{3. Results and Discussion}

\section{3.1 Fabrication and characterization of hMSC-only and hMSC/HUVEC spheroids}

254 96-well plates with cell-repellent surface were used to form spheroids composed of hMSC255 only and hMSC/HUVEC as described in our previous work [13]. As shown in Figure 1A, 256 optical microscopy images demonstrated that cell suspension with a density of 50,000 cells 257 per well was undergoing formation of a 3D cellular network, where cells coalesced into a 258 single spheroid along the bottom surface of the well plate. Once aggregation was completed, 259 spheroids slightly decreased in size, but progressively compacted over the course of culture

260 period (Figure 1A). All spheroids demonstrated a spherical and uniform shape at day 5 of 261 culture. The mean diameter of hMSC-only, and 92:8, 85:15, and 75:25 hMSC/HUVEC 262 spheroids was determined to be $\sim 694, \sim 655, \sim 649$, and $\sim 592 \mu \mathrm{m}$, respectively (Figure 1B). 263 Cell viability of spheroids at day 5 using LIVE/DEAD staining depicted $\sim 86$, and $\sim 92 \%$ for 
264 hMSC-only, and all hMSC/HUVEC spheroids respectively (Figure 1C). The cell viability of

265 all the groups introduced with HUVECs was found to be significantly higher than that of 266 hMSC-only group, which is in accordance to previous 2D co-culture reports [25]. In 267 particular, cell viability was uniform across the entire spheroid domain for HUVEC-involved 268 groups. H\&E staining images (Figure 1A) of cross-sections of spheroids confirmed evenly 269 distributed and well-connected cells with no significant difference in cell viability (Figure 270 1C) when comparing the number of HUVECs in hMSC spheroids. Also, immunofluorescent 271 staining of F-actin corroborated the results from the H\&E staining. hMSC/HUVEC spheroids 272 exhibited denser and more uniform cytoskeletal organization as compared to hMSC-only 273 spheroids, which exhibited lesser cellular density and empty space at the core. Moreover, 274 hMSC/HUVEC spheroids had significantly higher RNA amount. As shown in Figure 1D, the 275 RNA amount for hMSC-only, 92:8, 85:15, and 75:25 hMSC/HUVEC spheroids was 79, 276 126, 162, and $196 \mathrm{ng} /$ spheroid, respectively. Interestingly, the RNA amount of 277 hMSC/HUVEC spheroids decreased with increase in HUVEC concentration. This showed 278 that larger cellular spheroids needed to generate a higher amount of RNA for the maintenance 279 of cellular activities [26]. Thus, the dead empty core with reduced cell viability and RNA 280 amount in hMSC-only spheroid are a direct evidence to the role of HUVECs in enhancing the 281 crosstalk amongst hMSCs in the spheroids. Moreover, visibly more compaction, also 282 corroborated by the diameters of the spheroids, in the hMSC/HUVEC spheroids improved the 283 diffusion of the nutrients into the core of the spheroids and facilitated the removal of waste 284 products, enhancing the cell viability.

285 The surface tension of spheroids was measured as $495.8 \pm 30.2 \mathrm{mN} / \mathrm{m}$ for hMSC-only 286 spheroids, $661.9 \pm 127.1 \mathrm{mN} / \mathrm{m}, 865.7 \pm 73.8 \mathrm{mN} / \mathrm{m}$, and $940.6 \pm 98.8 \mathrm{mN} / \mathrm{m}$ for 92:8, 85:15, 287 and 75:25 hMSC/HUVEC spheroids respectively (Figure 1E). Notably, all hMSC/HUVEC 
288 groups showed increase in surface tension as compared to that of hMSC-only spheroid. The

$28975: 25$ group showed the highest surface tension amongst all groups, attributed to decrease in 290 diameter of hMSC/HUVEC spheroid with increase in HUVEC concentration, as discussed

291 earlier (Figure 1B). This shows that the compaction of the spheroids, depicted by the

292 decrease in diameter, is a direct influencer of the surface tension of the spheroids. The

293 increased compaction demonstrates that the hMSC/HUVEC spheroids are more in the

294 viscoelastic spectrum, i.e. more elastic (solid) than viscous (liquid), and hence possess

295 enhanced mechanical properties compared with hMSC-only spheroids.

296 In order to quantify the gene expression profiles of hMSC-only and hMSC/HUVEC

297 spheroids, mRNA expression of OCT4, PECAM-1, HIF1A, and COL6A1 genes was

298 investigated by RT-qPCR. As shown in Figure 1F, the mRNA expression of OCT4,

299 represented as pluripotency-associated gene [27], significantly upregulated for hMSC-only

300 and hMSC/HUVEC spheroids compared to conventional 2D culture condition of hMSC (2D

301 hMSC) and HUVEC (2D HUVEC) after five days of culture in growth media. Among all

302 groups, 92:8 hMSC/HUVEC spheroids showed the highest expression level of OCT4

303 compared to that for other groups. Additionally, all hMSC/HUVEC spheroids exhibited

304 higher PECAM-1 gene expression compared to 2D hMSC and hMSC-only spheroids.

305 PECAM-1 expression level was significantly increased with increase in HUVEC

306 concentration and 75:25 group exhibited the highest PECAM-1 expression. On the other

307 hand, the expression of HIF1A, which is a hypoxia-responsive transcription factor [28], was

308 significantly decreased with increase in HUVEC concentration. The highest expression level

309 of HIF1A was observed in hMSC-only spheroid. COL6A1 is a major structural component of

310 microfibrils in connective tissues and interact with other ECM components [29]. COL6A1

311 expression of hMSC-only and hMSC/HUVEC spheroids was significantly higher than that of 
312 2D hMSC and 2D HUVEC. Overall, all hMSC/HUVEC groups showed increased mRNA

313 expression of OCT4, PECAM, and COL6A1 and decreased HIF1A expression compared to

314 hMSC-only spheroids.

315 In summary, 92:8 hMSC/HUVEC spheroids showed higher cell viability and mechanical 316 properties (surface tension) compared to hMSC-only spheroids and demonstrated highest

317 RNA content and pluripotency potential. Based on these results, we showed that introduction

318 of only $8 \%$ HUVECs into the hMSCs is sufficient enough to enhance spheroid core cell

319 viability with improved mechanical properties. Therefore, to accomplish our goal of building

320 bone tissue and exploring the role of HUVECs in hMSCs spheroids on shape preservation of

321 3D constructs, we utilized 92:8 hMSC/HUVEC spheroids for the rest of the study, referred as

322 hMSC/HUVEC spheroid henceforth.

\section{$324 \quad 3.2$ Bioprinting of hMSC/HUVEC spheroids via AAB}

325 In our previous study, we developed an $\mathrm{AAB}$ platform for biofabrication of 3D constructs 326 with complex geometries [22]. This AAB system can precisely bioprint spheroids with a

327 diverse range of sizes (diameter $<80 \mu \mathrm{m}->800 \mu \mathrm{m}$ ) by picking up spheroids individually

328 and placing them onto desired locations on hydrogel-coated substrates. In this study, alginate

329 was printed onto glass substrates using a micro-valve dispenser, and partially cross-linked

330 using aerosol of $\mathrm{CaCl}_{2}$. hMSC/HUVEC spheroids were picked by a glass pipette and

331 bioprinted at desired locations onto the gel, according to previously determined patterns

332 (Figures 2A-C, Supplementary Video 1). This procedure was repeated for each of the

333 spheroids and after completion, the spheroids were stacked up in three layers to fabricate

334 each of the 3D complex-shaped constructs in $\mathrm{mm}$ scale, resembling a pyramid

335 (Supplementary Video 1), hexagon, and diamond topology. Owing to the inherent adhesive 
336 nature of hMSC/HUVEC spheroids ${ }^{21,}$ [22], they were well stacked up on the gel substrate

337 and maintained the 3D complex topologies without structural collapse.

338 After bioprinting, the constructs were overlaid with alginate using the micro-valve dispenser,

339 crosslinked with the aerosols of $\mathrm{CaCl}_{2}$, and then incubated for two days to facilitate fusion

340 amongst spheroids. As shown in Figure 2D, two days after bioprinting and incubation in

341 alginate, the $\sim 1 \mathrm{~mm}$ pyramid topography, assembled using hMSC/HUVEC spheroids,

342 maintained its defined shape with evidence of organized cytoskeletal filaments. After removal

343 of alginate and further incubation, the constructs were observed to undergo significant

344 compaction, lost their designed configuration and turned into tissue balls (Figure S1), which

345 is corroborated by previously published articles [14,22,30]. In order to direct stem cell

346 differentiation towards bone tissue formation, constructs composed of stem cells should be

347 inducted for about three weeks [14,16,31]. In this case, the bioprinted hMSC/HUVEC

348 construct deformed into a tissue aggregate within four days of being guided into bone tissue

349 differentiation. Hence, the concern with inherent compaction of spheroids leading to shape

350 contortion hinders the whole objective of bioprinting spheroids in complex desired

351 geometries. This led us to explore another strategy, by tuning the timelines of induction of

352 osteogenic differentiation, for retaining the geometry of constructs while bioprinting using 353 spheroids.

\section{$355 \quad 3.3$ Osteogenic differentiation of hMSC/HUVEC spheroids}

356 To induce osteogenesis, hMSC/HUVEC spheroids were cultured for five days in growth 357 media and the following 10 days in osteogenic media. The core of the spheroids was 358 visualized by $H \& E$ staining of the cross-sections. As shown in Figures 3A1 and 3B1, 359 hMSC/HUVEC spheroids, after induction to osteogenic lineage, showed more compact and 
360 condensed structures with uniformly distributed cells compared to that in hMSC-only

361 spheroids. Mainly, this phenomenon is more pronounced at the center of spheroids, with ring

362 like structures at the periphery of the spheroids. This is due to the presence of HUVECs in

363 cellular spheroids [16]. Osteogenic cells begin the deposition of mineral deposits when they

364 differentiate into osteoblasts [32]. Calcium deposition of hMSC-only and hMSC/HUVEC

365 spheroids was confirmed by alizarin red S (ARS) staining (Figures 3A2 and 3B2). The

366 deposited calcium amount of hMSC/HUVEC spheroid was substantially higher than that of

367 hMSC-only spheroid with uniform calcium deposition across the entire cross-section, except

368 for the periphery of the spheroid. The presence of the ring like structure (H\&E) and absence

369 of calcium deposition (ARS) at the periphery of the hMSC/HUVEC spheroids led us to

370 characterize the localization of the hMSCs and HUVECs in the spheroids after osteogenic

371 induction. RUNX2, an early osteogenic marker, was verified by immunocytochemical

372 staining along with CD31 staining to confirm the cells of endothelial lineage. RUNX2 marker

373 in hMSC/HUVEC spheroids was uniformly distributed across the cross-section of the

374 spheroid while lesser expression of RUNX2 was observed in hMSC-only spheroid (Figures

375 3A3 and 3B3). Besides, hMSC/HUVEC spheroids showed more endotheliogenic-specific

376 marker than hMSC-only spheroids, as indicated by positive staining of CD31, with more

377 localized CD31+ cells at the periphery of the spheroid, forming a ring like structure, which is

378 in agreement with previous findings [33]. Thus, hMSC/HUVEC spheroids demonstrated

379 enhanced proliferation and increased mineralization which is direct evidence of enhanced

380 osteogenic potential due to a synergistic display between the two cell types [34,35]. Crosstalk

381 between hMSCs and endothelial cells is not only dependent on microenvironmental diffusion

382 factors but also, on gap junction alpha-1 protein [36] which demonstrates the necessity of

383 direct cell-cell contact between hMSCs and HUVECs [37]. Besides this juxtacrine 
384 mechanisms by the gap junctional activity, endothelial cells mediated paracrine factors

385 including vascular endothelial growth factor (VGEF) and the inflammatory mediator

386 prostaglandin $\mathrm{E}_{2}$ (PGE2) augments the crosstalk between periodontal ligament stem cells

387 (PDLSCs) and endothelial cells. This, in turn, enhances the osteogenic potential of PDLSCs

388 under hypoxia regulated by mitogen-activated protein kinase (MAPK) kinase/extracellular

389 signal-regulated kinase and p38 MAPK pathways [18]. As the core of the spheroids are

390 deemed more hypoxic compared to the periphery, osteogenic differentiation of stem cells is

391 enhanced by the $\mathrm{PGE}_{2}$ and VEGF factors secreted by the HUVECs. HUVECs have also

392 shown to express bone morphogenic protein 2, which induces osteogenic differentiation of

393 stromal stem cells [38]

394 With considerable qualitative evidence demonstrating the effects of HUVECs on osteogenic

395 potential of hMSCs, we also analyzed the mRNA expression of type I collagen (COL1),

396 alkaline phosphatase (ALP), and bone sialoprotein (BSP) to quantify the same. All the

397 experimental groups consisting of 2D hMSC, hMSC-only spheroids and hMSC/HUVEC

398 spheroids were divided into two categories - cultured in either the maintenance media

399 (labeled as "Non-osteo") or osteogenic media (labeled as "Osteo"). As shown in Figure 3C,

400 all groups cultured in osteogenic media exhibited higher expression of COL1, ALP, and BSP

401 than those in maintenance media. The early-stage osteogenic differentiation markers of COL1

402 and ALP increased in the order of 2D hMSC, hMSC-only, and hMSC/HUVEC in both types

403 of culture media. COL1 expression for hMSC-only and hMSC/HUVEC spheroids was

404 significantly higher than that for 2D hMSC. This demonstrates the efficacy of 3D spheroids

405 to accurately demonstrate the influence of microenvironmental factors on osteogenic

406 differentiation ${ }^{22}$. Also, hMSC/HUVEC spheroids cultured in osteogenic media exhibited

407 significantly increased ALP expression compared to both 2D hMSC and hMSC-only 
408 spheroids cultured similarly. This is a direct evidence that the endothelial cells enhance the

409 differentiation of hMSCs. Although, the precise role of HUVECs on osteogenic

410 differentiation is debated, many 2D co-cultures with hMSCs and HUVECs have reported

411 such observations [39,40]. The expression of BSP, a late osteogenic differentiation marker,

412 significantly increased in the order of 2D hMSC, hMSC-only, and hMSC/HUVEC spheroids

413 similar to the COL1 and ALP results. In particular, 3D hMSC/HUVEC showed the highest

414 expression of BSP compared to other groups. Overall, it was found that hMSC/HUVEC

415 spheroids exhibited enhanced early and late stage osteogenic differentiation, both in presence

416 and absence of osteogenic media corroborating our earlier H\&E, ARS and

417 immunocytochemical staining and previous literature [39,40].

418 Therefore, hMSC/HUVEC spheroids were used for bioprinting of osteogenic tissues.

419 Quiescence is inherently associated with niche resident stem cells. Previously studies have 420 reported lower hMSCs proliferation rates in a co-culture system with HUVECs [33], 421 suggesting the role of HUVECs in a retardation of hMSCs activity. Inducing the

$422 \mathrm{hMSC} / \mathrm{HUVEC}$ spheroids to differentiation media before bioprinting lowers the proliferative 423 potential of hMSCs and allow the spheroids to be in their osteogenic differentiation pathway 424 [41]. Hence, in this study, we utilized mid-term osteogenic hMSC/HUVEC spheroids for 425 bioprinting scaffold-free bone-tissue constructs with an expectation of reduced deformation 426 in the bioprinted geometry.

\section{$428 \quad 3.43 \mathrm{D}$ bioprinting of osteogenic tissues}

429 To demonstrate the ability of osteogenic hMSC/HUVEC spheroids to serve as building 430 blocks in bioprinting, different topographies resembling triangle, hexagon, and diamond in a 431 single layer were bioprinted using the AAB system. HMSC/HUVEC spheroids cultured for 
432 five days in maintenance media and differentiated for 10 days in osteogenic media were

433 bioprinted on the gel substrate, overlaid with the sacrificial alginate, and then incubated for

434 another two days in the osteogenic media. As shown in Figure 4A, hMSC/HUVEC spheroids

435 were precisely arranged into a diamond shape and after incubation, fused into a single patch

436 of tissue. We also demonstrated a diamond shaped topography in three layers with osteogenic

437 hMSC/HUVEC spheroids (Figure 4B). Bioprinted single-layered osteogenic tissue construct

438 exhibited high cell viability from the periphery to the core of the spheroid with a negligible

439 number of dead cells, as indicated by LIVE/DEAD staining. In histological analysis, H\&E-

440 stained tissue sections revealed that osteogenic hMSC/HUVEC spheroids were tightly self-

441 assembled to each other, and bioprinted tissues maintained their original shape without

442 falling apart. The histological analysis of the cross-sections of the 3D diamond-shaped

443 construct by $H \& E$ presented uniform cell distribution through the entire 3D structure with a

444 very well-fused interface amongst the spheroids. Mineralization was confirmed by measuring

445 mineralized volume and calibrated bone mineral density by $\mu \mathrm{CT}$ analysis. The results showed

446 that the single-layered bioprinted constructs were predominantly composed of mineralized

447 matrix, which was indicated by values exceeding the threshold range of $200 \mathrm{mgHA} / \mathrm{ccm}$. The

448 mineralized volume in the single-layered triangle, hexagon and diamond shaped

449 configurations was measured to be $0.051,0.056$ and $0.081 \mathrm{~mm}^{3}$, respectively. Uniform

450 mineralization was also observed in the 3-layered diamond topography, with no significant

451 alternation in its shape. The mineralized volume in first, second- and third-layer of the 3-

452 layered geometry was measured at $0.163,0.036$ and $0.004 \mathrm{~mm}^{3}$, respectively.

453 Thus, in this study, we presented a new approach to fabricating scaffold-free bone tissue 454 constructs using the $\mathrm{AAB}$ system with mid-term osteogenic hMSC/HUVEC spheroids as 
455 building blocks. The constructs retained the bioprinted shape and demonstrated uniform

456 mineralization throughout the entire 3D configuration.

457 This practical AAB approach could be refined in the near-future to enhance the throughput

458 and scalability and has the likelihood to be used as a reproducible tissue bioprinting platform,

459 for various applications, including organ-on-a-chip devices, microfluidics, organoid

460 engineering and tissue engineering. In addition, since lack of vascularization is one of the

461 major roadblocks for functional bone tissue constructs[42] leading to tissue necrosis, long-

462 term bone tissue viability has to be addressed in the future by the fabrication of scaffold-free

463 constructs with an extensive and efficient network of vascular micro-vessels is desirable,

464 prior to preclinical testing.

\section{4. Conclusion}

466 In conclusion, we present an effective spheroid bioprinting strategy using the AAB system,

467 which facilitates bioprinting of osteogenic hMSC/HUVEC spheroids to fabricate scaffold-

468 free 3D bone tissue constructs. This study addresses the limitations of the previously explored

469 spheroid bioprinting techniques by enabling precise positioning of spheroids onto a hydrogel

470 (11\% with respect to the spheroid diameter), preservation of the geometrical shape of the

471 fabricated construct by reducing compaction, and induction of uniform bone formation across

472 the entire spheroid. HUVECs were introduced into hMSCs to investigate their influence on

473 spheroid formation and compaction, osteogenic differentiation and reduction of shape

474 deformation after differentiation. Our findings demonstrate that osteogenically-differentiated

475 hMSC/HUVEC spheroids, with as little as $8 \%$ of HUVECs, could be used as building blocks

476 for bone tissue fabrication. These spheroids demonstrated reduced necrosis, increased cell

477 viability in the core of the spheroid, enhanced differentiation into osteogenic lineage, and

478 improved mechanical properties. Additionally, our bioprinting strategy exploited the cohesive 
479 nature of spheroids and reduced the proliferative potential of hMSCs after differentiation; this

480 enabled building a scaffold-free 3D construct without any major shape change after

481 bioprinting. Such a bioprinting approach, to facilitate fabrication of 3D geometries with

482 negligible shape changes, using spheroids composed of differentiated stem cells and

483 introduced with endothelial cells to enhance cell viability and differentiation, provides a new

484 direction in bottom-up, scaffold-free bone tissue fabrication.

\section{Acknowledgement}

487 This work has been supported by National Science Foundation Awards 1914885 and a

488 Convergence grant from the Materials Research Institute at Penn State University. D.N. H.

489 acknowledges the support from the National Research Foundation of Korea (NRF) grant

490 funded by the Korean Government (MIST) (2017R1D1A1B04030398)

491 (2020R1C1C1007129).

492

493 Contributions

494 D.N.H., B.A. and I.T.O. designed the research. D.N.H., B.A., M.D., and H.W. performed the 495 experiments, and D.N.H., B.A. D.B., G.L., and I.T.O. evaluated the results. All authors 496 contributed to writing of the manuscript and agreed the final content of the manuscript.

498 Data availability: All data needed to evaluate the conclusions in the paper are present in the 499 paper and/or the Supplementary Materials. Additional data related to this paper may be 500 requested from the authors.

501

502 6. References 
503 [1] Amini A R, Laurencin C T and Nukavarapu S P 2012 Bone Tissue Engineering:

[3] Hutmacher D W 2000 Scaffolds in tissue engineering bone and cartilage Biomaterials 21 2529-43

[4] Datta P, Ozbolat V, Ayan B, Dhawan A and Ozbolat I T 2017 Bone tissue bioprinting for craniofacial reconstruction Biotechnol. Bioeng. 114 2424-31

[5] Hung B P, Naved B A, Nyberg E L, Dias M, Holmes C A, Elisseeff J H, Dorafshar A $\mathrm{H}$ and Grayson W L 2016 Three-Dimensional Printing of Bone Extracellular Matrix for Craniofacial Regeneration ACS Biomater. Sci. Eng. 2 1806-16

[6] Liu X and Ma P X 2004 Polymeric Scaffolds for Bone Tissue Engineering Ann. Biomed. Eng. 32 477-86

[7] Burg K J L, Porter S and Kellam J F 2000 Biomaterial developments for bone tissue engineering Biomaterials 21 2347-59

[8] Atala S V M \& A 2014 3D Bioprinting of Tissues and Organs Nat. Biotechnol. 32 773-85

[9] Rather H A, Jhala D and Vasita R 2019 Dual functional approaches for osteogenesis coupled angiogenesis in bone tissue engineering Mater. Sci. Eng. C 103109761

[10] Jørgensen N R, Henriksen Z, Sørensen O H and Civitelli R 2004 Dexamethasone, BMP-2, and 1,25-dihydroxyvitamin D enhance a more differentiated osteoblast phenotype: validation of an in vitro model for human bone marrow-derived primary osteoblasts Steroids 69 219-26

[11] Ozbolat I T 2015 Scaffold-Based or Scaffold-Free Bioprinting: Competing or Complementing Approaches? J. Nanotechnol. Eng. Med. 6024701

[12] Langenbach F, Naujoks C, Smeets R, Berr K, Depprich R, Kübler N and Handschel J 2013 Scaffold-free microtissues: differences from monolayer cultures and their potential in bone tissue engineering Clin. Oral Investig. 17 9-17

[13] Peng W, Datta P, Wu Y, Dey M, Ayan B, Dababneh A and Ozbolat I T 2018 Challenges in Bio-fabrication of Organoid Cultures Advances in Experimental Medicine and Biology pp 53-71

[14] Fennema E, Rivron N, Rouwkema J, van Blitterswijk C and de Boer J 2013 Spheroid culture as a tool for creating 3D complex tissues Trends Biotechnol. 31 108-15

[15] Laschke M W, Schank T E, Scheuer C, Kleer S, Shadmanov T, Eglin D, Alini M and Menger M D 2014 In vitro osteogenic differentiation of adipose-derived mesenchymal stem cell spheroids impairs their in vivo vascularization capacity inside implanted porous polyurethane scaffolds Acta Biomater. 10 4226-35

[16] Heo D N, Hospodiuk M and Ozbolat I T 2019 Synergistic interplay between human MSCs and HUVECs in 3D spheroids laden in collagen/fibrin hydrogels for bone tissue engineering Acta Biomater. 95 348-56

[17] Cerwinka W H, Sharp S M, Boyan B D, Zhau H E, Chung L W K and Yates C 2012 Differentiation of human mesenchymal stem cell spheroids under microgravity conditions Cell Regen.

[18] Wu Y, Cao H, Yang Y, Zhou Y, Gu Y, Zhao X, Zhang Y, Zhao Z, Zhang L and Yin J 2013 Effects of vascular endothelial cells on osteogenic differentiation of noncontact co-cultured periodontal ligament stem cells under hypoxia J. Periodontal Res. $4852-$ 65

[19] Moldovan N I, Hibino N and Nakayama K 2017 Principles of the Kenzan Method for 
Robotic Cell Spheroid-Based Three-Dimensional Bioprinting Tissue Eng. Part B Rev. 23 237-44

[20] Norotte C, Marga F S, Niklason L E and Forgacs G 2009 Scaffold-free vascular tissue engineering using bioprinting Biomaterials 30 5910-7

[21] Mironov V, Visconti R P, Kasyanov V, Forgacs G, Drake C J and Markwald R R 2009 Organ printing: Tissue spheroids as building blocks Biomaterials 30 2164-74

[22] Ayan B, Heo D N, Zhang Z, Dey M, Povilianskas A, Drapaca C and Ozbolat I T 2020 Aspiration-assisted bioprinting for precise positioning of biologics $S c i . A d v .6$ eaaw5111

[23] Hospodiuk M, Dey M, Ayan B, Sosnoski D, Moncal K K, Wu Y and Ozbolat I T 2018 Sprouting angiogenesis in engineered pseudo islets Biofabrication 10035003

[24] Mashiatulla M, Ross R D and Sumner D R 2017 Validation of cortical bone mineral density distribution using micro-computed tomography Bone 99 53-61

[25] Bidarra S J, Barrias C C, Barbosa M A, Soares R, Amédée J and Granja P L 2011 Phenotypic and proliferative modulation of human mesenchymal stem cells via crosstalk with endothelial cells Stem Cell Res. 7 186-97

[26] Marguerat S and Bähler J 2012 Coordinating genome expression with cell size Trends Genet. 28 560-5

[27] Matic I, Antunovic M, Brkic S, Josipovic P, Caput Mihalic K, Karlak I, Ivkovic A and Marijanovic I 2016 Expression of OCT-4 and SOX-2 in Bone Marrow-Derived Human Mesenchymal Stem Cells during Osteogenic Differentiation Open Access Maced. J. Med. Sci. 49

[28] Anada T, Fukuda J, Sai Y and Suzuki O 2012 An oxygen-permeable spheroid culture system for the prevention of central hypoxia and necrosis of spheroids Biomaterials 33 8430-41

[29] Gara S K, Grumati P, Urciuolo A, Bonaldo P, Kobbe B, Koch M, Paulsson M and Wagener R 2008 Three Novel Collagen VI Chains with High Homology to the $\alpha 3$ Chain J. Biol. Chem. 283 10658-70

[30] Laschke M W and Menger M D 2017 Life is 3D: Boosting Spheroid Function for Tissue Engineering Trends Biotechnol.

[31] Vrij E, Rouwkema J, LaPointe V, van Blitterswijk C, Truckenmüller R and Rivron N 2016 Directed Assembly and Development of Material-Free Tissues with Complex Architectures Adv. Mater. 28 4032-9

[32] Beck G R 2003 Inorganic phosphate as a signaling molecule in osteoblast differentiation J. Cell. Biochem. 90 234-43

[33] Saleh F, Whyte M and Genever P 2011 Effects of endothelial cells on human mesenchymal stem cell activity in a three-dimensional in vitro model Eur. Cells Mater. 22 242-57

[34] Kuss M A, Wu S, Wang Y, Untrauer J B, Li W, Lim J Y and Duan B 2018 Prevascularization of 3D printed bone scaffolds by bioactive hydrogels and cell coculture J. Biomed. Mater. Res. Part B Appl. Biomater. 106 1788-98

[35] Stoppato M, Stevens H Y, Carletti E, Migliaresi C, Motta A and Guldberg R E 2015 Influence of scaffold properties on the inter-relationship between human bone marrow derived stromal cells and endothelial cells in pro-osteogenic conditions Acta Biomater. 25 16-23

[36] Villars F, Guillotin B, Amédée T, Dutoya S, Bordenave L, Bareille R and Amédée J 2002 Effect of HUVEC on human osteoprogenitor cell differentiation needs heterotypic gap junction communication Am. J. Physiol. Physiol. 282 C775-85 
599 [37] Grellier M, Ferreira-Tojais N, Bourget C, Bareille R, Guillemot F and Amédée J 2009 Role of vascular endothelial growth factor in the communication between human

\section{Ethics declarations}

622 The authors declare no competing interests. 

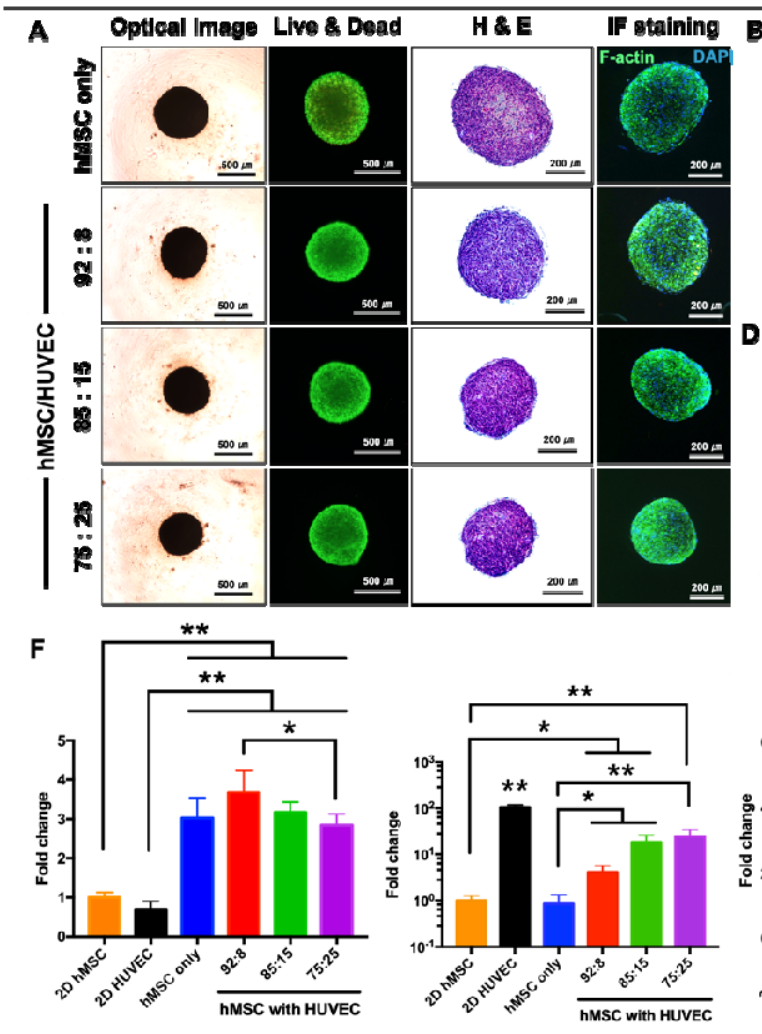

OCT4

PECAM
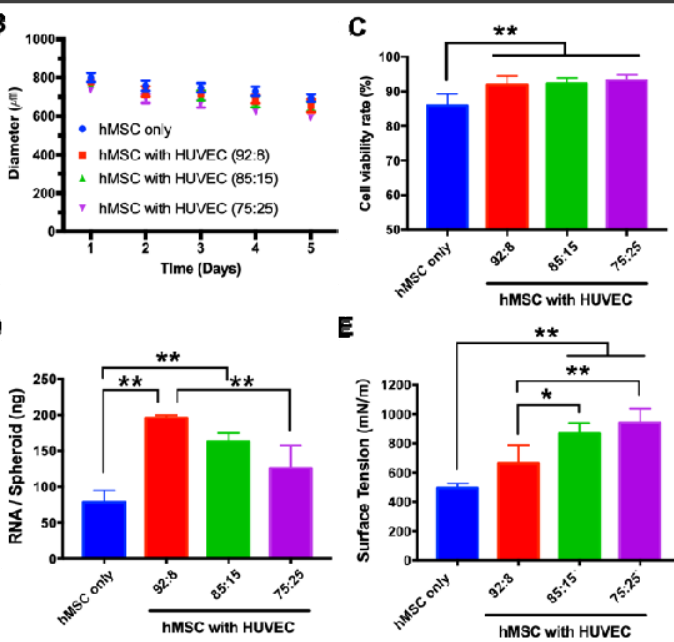

E

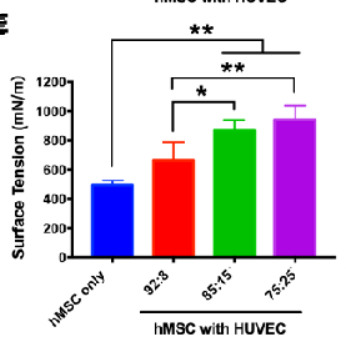

630 Figure 1. Formation of 3D spheroids and their characterization five days after incubation:

631 (A) optical, LIVE/DEAD assay, histology, and F-actin staining images of hMSC-only and

632 hMSC/HUVEC spheroids, (B) compaction of the spheroids over time, determined from the

633 reduction in their spheroid diameter. (C) cell viability, quantified from LIVE/DEAD assay

634 (D) quantification of RNA concentration using Nanodrop (E) surface tension (F) gene

635 expression measurements for pluripotent stem cell (OCT4), endothelial cell (PECAM),

636 hypoxia (H1F1A), and ECM (COL6A1) related markers in 2D hMSC, hMSC-only and

637 hMSC/HUVEC spheroids; *p $<0.05$ and **p $<0.01$. We showed that introduction of only

$6388 \%$ HUVECs into the hMSCs is sufficient enough to enhance cell viability at the core of the

639 spheroid with improved mechanical properties. 


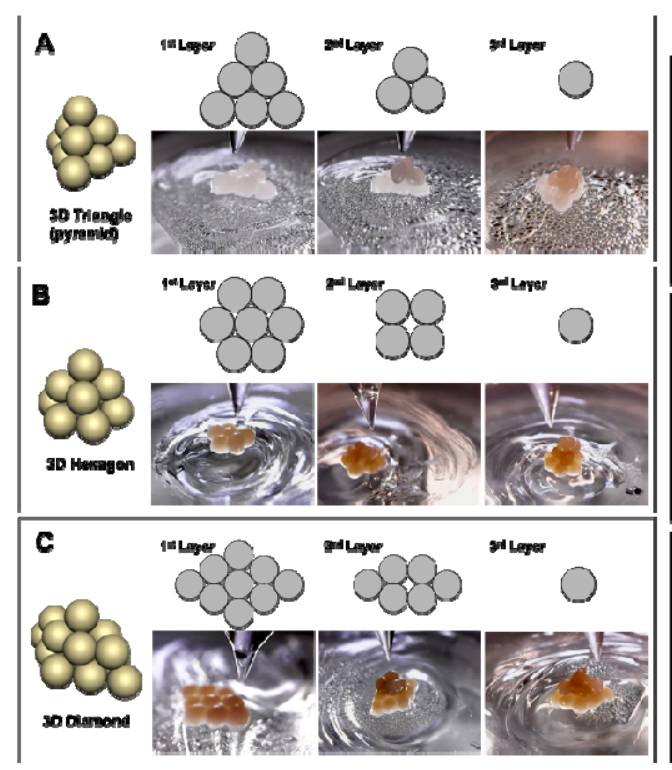

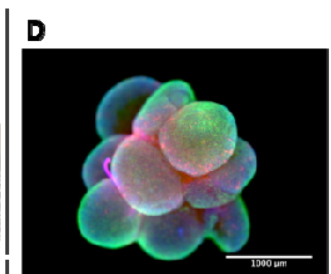

\section{From the side}
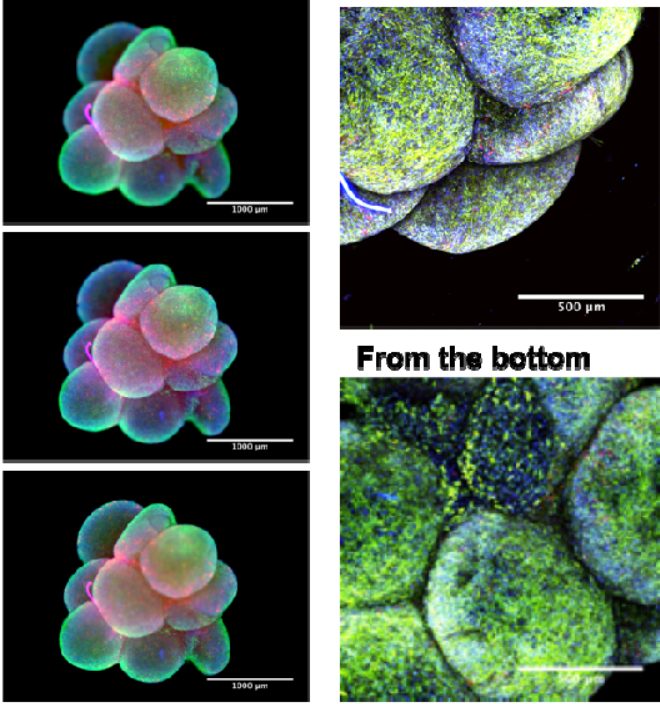

From the bottom

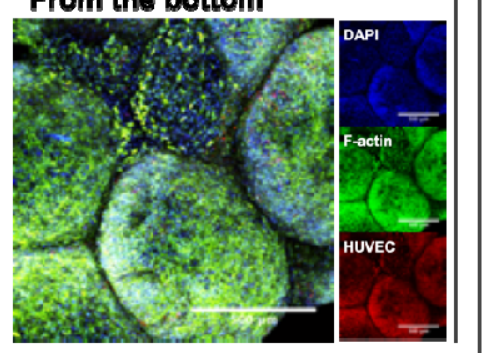

641 Figure 2. 3D Bioprinting of hMSC/HUVEC spheroids via Aspiration-assisted Bioprinting.

642 Schematic diagrams and real-time photographs of a 3D pyramid (A), 3D hexagon (B), and

643 3D diamond (C) topographies using spheroids as building blocks. (D) Fluorescent images of

644 bioprinted pyramid of hMSC/ ddTomato $^{+}$HUVEC spheroids, which were stained with DAPI

645 and F-actin. Confocal images of pyramid constructs (side and bottom views). 


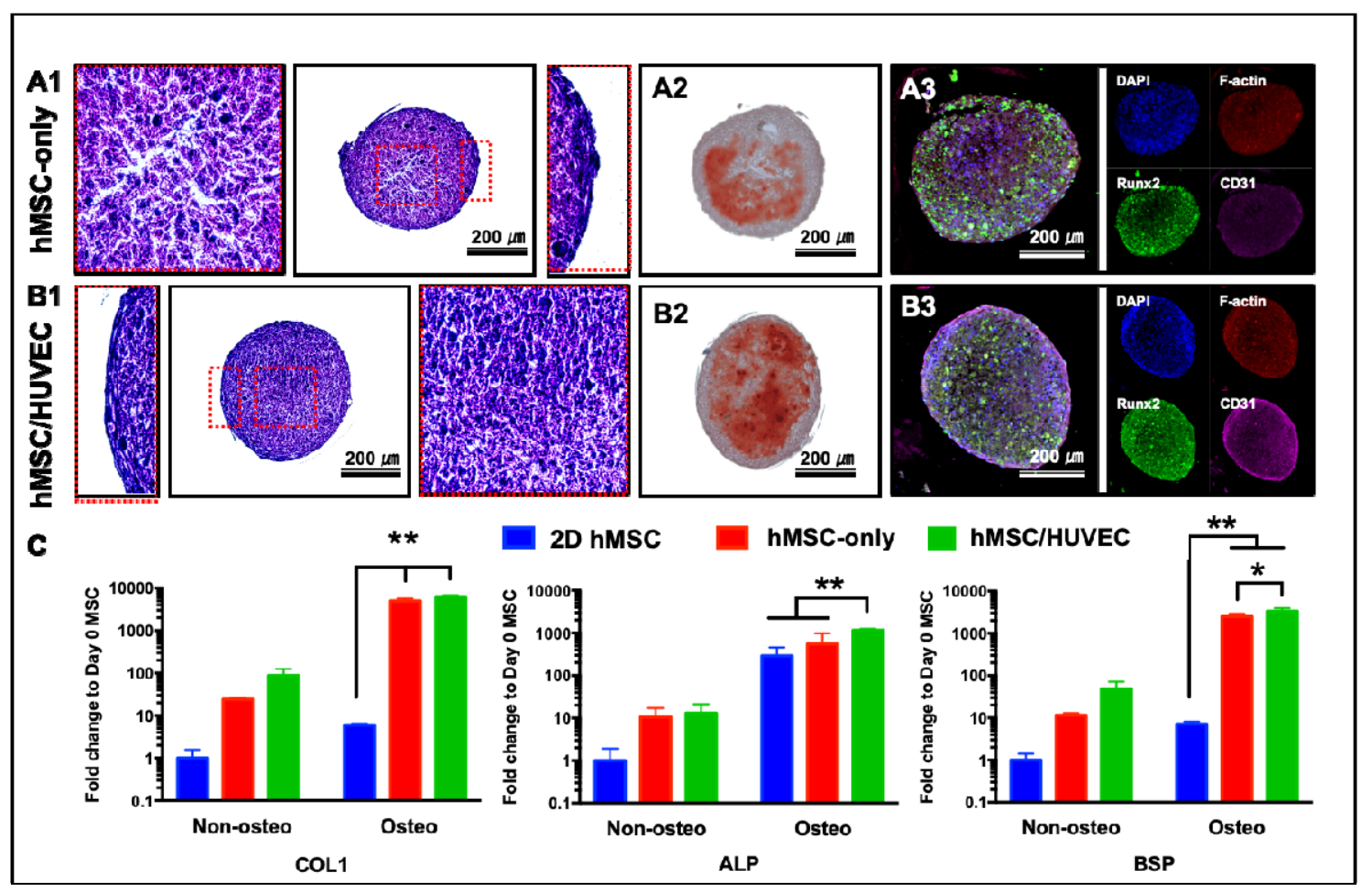

653 Figure 3. Characterization of osteogenically differentiated hMSC/HUVEC spheroids,

654 cultured for five days in growth media and 10 days in osteogenic media: Representative 655 images of (A1- B1) H\&E staining, (A2-B2) ARS staining, and (A3- B3) IF staining with 656 RUNX2 (Green), F-actin (Red), CD31 (Magenta), and cell nuclei (Blue) of hMSC-only and 657 hMSC/HUVEC spheroids respectively. (C) Osteogenic related gene expression levels of 2D 658 hMSC, hMSC-only, and hMSC/HUVEC spheroids cultured in growth and osteogenic media; $659 * \mathrm{p}<0.05$ and $* * \mathrm{p}<0.01$. The results demonstrate the efficacy of HUVECs incorporation in 660 hMSC spheroids to enhance osteogenic differentiation across the entire spheroid domain. 


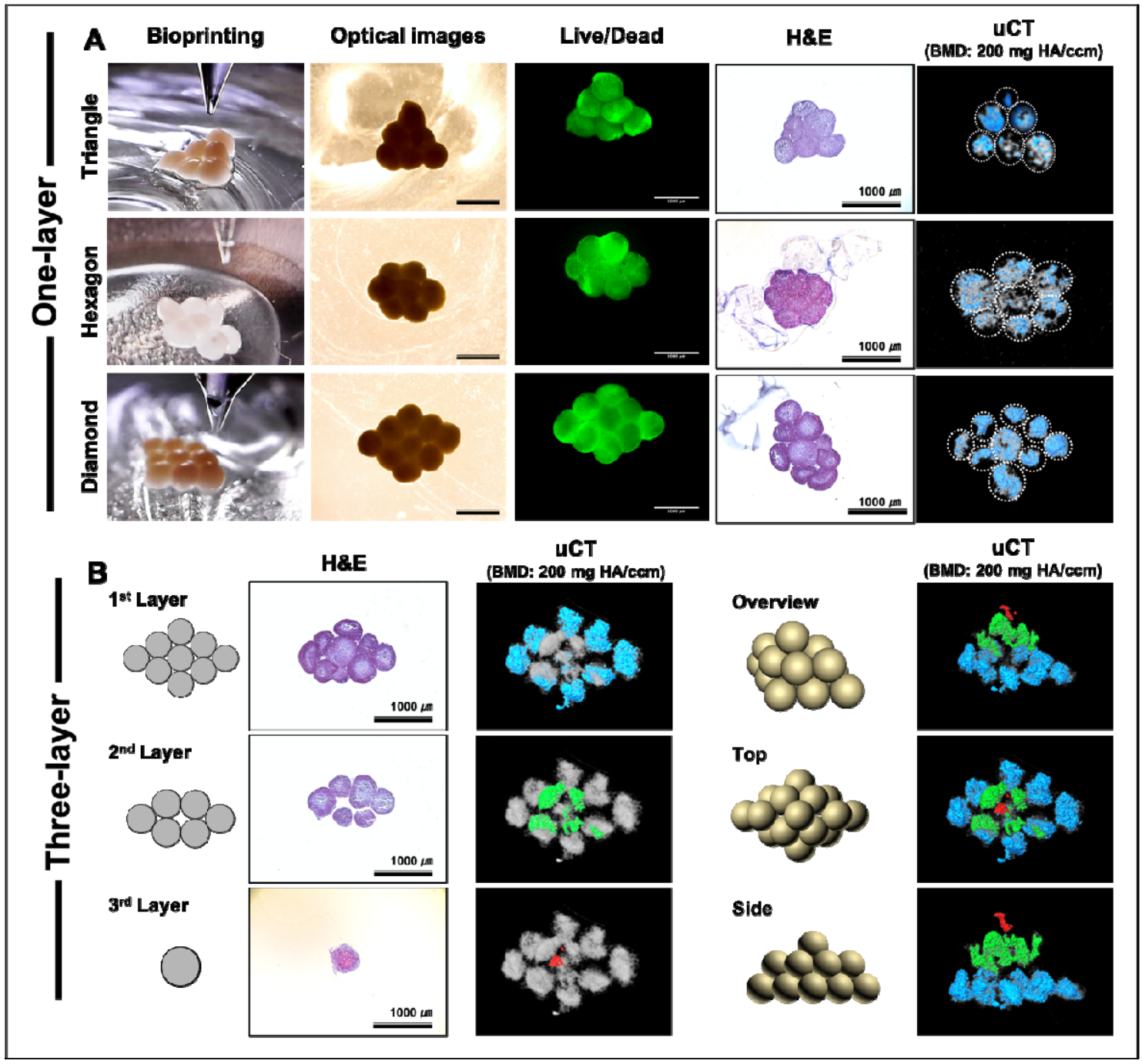

667 Figure 4. 3D biofabrication of (A) triangle, hexagon, and diamond-shaped topographies in a

668 single layer (A) and 3 layers (B) using mid-term osteogenic differentiated hMSC/HUVEC

669 spheroids. The bioprinted tissue constructs were characterized by optical microscopy,

670 LIVE/DEAD assay, $\mathrm{H} \& \mathrm{E}$ and $\mu \mathrm{CT}$ showing bone formation and mineralization two days

671 post bioprinting. The red, green and blue colors in the overview uCT scans depict the $3^{\text {rd }}, 2^{\text {nd }}$

672 and $1^{\text {st }}$ layer, respectively, of the 3-layered diamond-shaped bioprinted tissue. We show 673 negligible shape changes in the 3D tissue constructs post-bioprinting utilizing pre674 differentiated hMSC/HUVEC spheroids. 
679 Figure S1. Post-bioprinting, hMSC/ tdTomato ${ }^{+}$HUVEC tissue constructs were induced to

680 osteogenic differentiation. The tissue constructs lost their shape and turned into tissue balls

681 over a function of time (D: days). 